\title{
On the mesoscopic origins of high viscosities in some polyelectrolyte- surfactant mixtures
}

Cite as: J. Chem. Phys. 143, 074902 (2015); https://doi.org/10.1063/1.4928583

Submitted: 16 March 2015 . Accepted: 08 July 2015. Published Online: 18 August 2015

Ingo Hoffmann, Bela Farago, Ralf Schweins, Peter Falus, Melissa Sharp, Sylvain Prévost (D), and Michael Gradzielski

\section{ARTICLES YOU MAY BE INTERESTED IN}

Structure and dynamics of polyelectrolyte surfactant mixtures under conditions of surfactant excess

The Journal of Chemical Physics 145, 124901 (2016); https://doi.org/10.1063/1.4962581

Dynamics of small unilamellar vesicles

The Journal of Chemical Physics 148, 104901 (2018); https://doi.org/10.1063/1.5009424

Effect of interlamellar interactions on shear induced multilamellar vesicle formation

The Journal of Chemical Physics 147, 034905 (2017); https://doi.org/10.1063/1.4994563

\section{Lock-in Amplifiers up to $600 \mathrm{MHz}$}
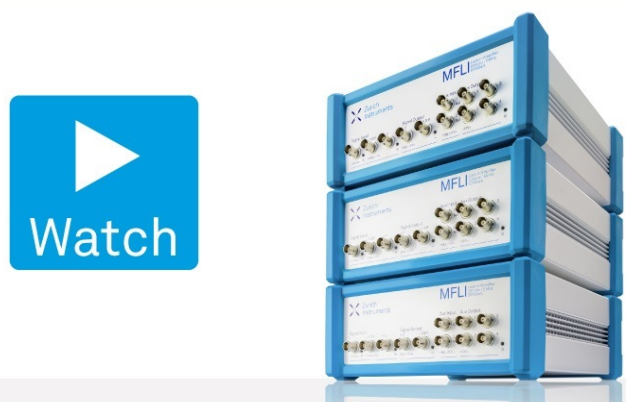

J. Chem. Phys. 143, 074902 (2015); https://doi.org/10.1063/1.4928583 


\title{
On the mesoscopic origins of high viscosities in some polyelectrolyte-surfactant mixtures
}

\author{
Ingo Hoffmann, ${ }^{1,2, a)}$ Bela Farago, ${ }^{2}$ Ralf Schweins, ${ }^{2}$ Peter Falus, ${ }^{2}$ Melissa Sharp, ${ }^{2}$ \\ Sylvain Prévost, ${ }^{1,3, b)}$ and Michael Gradzielski ${ }^{1, c)}$ \\ ${ }^{1}$ Stranski-Laboratorium für Physikalische und Theoretische Chemie, Institut für Chemie, Technische Universität \\ Berlin, Straße des 17. Juni 124, Sekr. TC 7, D-10623 Berlin, Germany \\ ${ }^{2}$ Institut Max von Laue-Paul Langevin (ILL), F-38042 Grenoble Cedex 9, France \\ ${ }^{3}$ Helmholtz-Zentrum Berlin, D-14109 Berlin, Germany
}

(Received 16 March 2015; accepted 8 July 2015; published online 18 August 2015)

\begin{abstract}
Oppositely charged polyelectrolyte (PE) surfactant mixtures allow the control of rheological parameters of a solution even at fairly low concentrations. For example, addition of $0.3 \mathrm{wt}$ \% of anionic surfactant to a 1 wt. \% solution of the polycation JR 400 increases the viscosity by 4 orders of magnitude. Recently, we could show that this increase is related to the formation of mixed, rod-like PE/surfactant aggregates which interconnect several polyelectrolyte chains [Hoffmann et al., Europhys. Lett. 104, 28001 (2013)]. In this paper, we refine our structural model of the aggregates to obtain a more consistent picture of their internal structure for different anionic surfactants. Combining small angle neutron scattering (SANS) and neutron spin-echo (NSE) allows us to determine the size of the aggregates. By comparing different contrasts, the internal structure of the aggregates can be elucidated and it is seen that the PE in the aggregates retains a relatively high freedom of movement. We proceeded to investigate the influence of the surfactant concentration and the surfactant type on structure and dynamics of the mixed aggregates. It is seen that the structural parameters of the aggregates depend very little on the surfactant concentration and headgroup. However, it is crucial to incorporate a sufficient amount of PE in the aggregates to increase the viscosity of the aggregates. By comparing viscous samples at $1 \mathrm{wt}$ \% PE concentration with samples at a PE concentration of 0.3 wt. $\%$, where no significant increase in viscosity is observed, we find that similar aggregates are formed already at this lower PE concentrations. However, the amount of PE incorporated in them is insufficient to interconnect several PE chains and therefore, they do not increase viscosity. So, our detailed investigation combining contrast variation SANS and NSE does not only allow to explain the viscosity behavior but also to deduced detailed information regarding the structures and the dynamics especially of the polyelectrolyte within the complexes. (C) 2015 AIP Publishing LLC. [http://dx.doi.org/10.1063/1.4928583]
\end{abstract}

\section{INTRODUCTION}

Oppositely charged polyelectrolyte (PE) surfactant systems have gained quite some interest over the past few decades. They can self assemble in a number of different structures, depending on several parameters such as the concentration of the components and their chemical nature which in turn determine parameters such as the backbone rigidity of the PE which consists of an intrinsic and an electrostatic part, ${ }^{1-4}$ the surfactant head group, the length and diameter of the hydrophobic part of the surfactant, and the contour length of the PE and its charge density.

These mixtures form complexes already at very low concentrations and the aggregation is usually driven by the release of counterions. Mixing equimolar amounts of charges, precipitation is observed. However, if an excess of either surfactant or PE charges is present, stable, clear solutions are formed

\footnotetext{
a)ingo.hoffmann@tu-berlin.de

b) Present address: ESRF-The European Synchrotron, CS40220, F-38043 Grenoble Cedex 9, France.

c) michael.gradzielski@tu-berlin.de
}

where the precise amount of excess charge required (the phase boundary) may vary largely from system to system..$^{5-32}$

Fundamental scientific interest aside, such mixtures have a number of applications, e.g., in cosmetics, detergency, drug delivery, and oil recovery. ${ }^{5,33-36}$

In particular, mixtures of the cationically modified, cellulose based PE JR 400 and several anionic surfactants showed a remarkable increase of viscosity by up to 4 orders of magnitude if the concentration of the PE was high enough, typically on the order of $0.5 \mathrm{wt} . \%$; such mixtures and similar systems have been investigated previously to quite some extent. ${ }^{37-47}$ While the viscosity is maximal at an excess of PE charges just next to the phase boundary, the enhancement in viscosity is lost if an excess of surfactant is added, i.e., when the precipitate becomes dissolved again by surfactant. It might be noted that a similar viscosity increase has been observed for the cationic cellulose ether JR 400 upon addition of anionic polymers. ${ }^{48}$

In a recent investigation, ${ }^{49}$ we could relate the increased viscosity to the formation of mixed rod-like aggregates, the structure of which could be elucidated with small angle neutron scattering (SANS) and neutron spin-echo (NSE) experiments. 
In particular, NSE allowed to determine the length of the aggregates, which proved to be difficult using only SANS, as the onset of the Guinier regime of the rod-like aggregates is obscured by a structure peak. Additionally, it could be shown that the PE in the aggregates retains its freedom of movement to a significant part and is not frozen in them.

However, so far, a detailed relation between the observed changes of the rheological properties and the mesoscopic structure is still missing. Therefore, we investigate here the effect of PE and surfactant concentration and of different surfactant types on the structure, composition, and dynamics of the aggregates using SANS and NSE, taking advantage of the unique ability of neutron scattering to vary the contrast by simply changing the isotopic composition of the sample and thereby rendering different parts of the structure visible. By doing so, we aim at gaining a better understanding of the relation between mesoscopic structure and macroscopic viscosity and to derive a self-consistent picture of structure and dynamics in such systems.

\section{MATERIALS AND METHODS}

SANS experiments were performed on the instruments D11 at the Institut Laue Langevin (ILL) in Grenoble, France and the instrument V4 at the Helmholtz Zentrum Berlin (HZB). Measurements on D11 were done at a wavelength $\lambda$ of $6 \AA$ and for sample-to-detector distances of 1.2, 8 , and $34 \mathrm{~m}$ to cover a range of the magnitude of the scattering vector $Q$ from 0.018 to $51 / \mathrm{nm}(Q=4 \pi / \lambda \sin (\theta / 2)$, with scattering angle $\theta)$ while measurements on $\mathrm{V} 4$ were performed at a wavelength of $4.57 \AA$ at sample to detector distances of $0.84,3.83$, and $14.83 \mathrm{~m}$ to cover a $Q$-range from 0.06 to $7.61 / \mathrm{nm}$. Transmissions were measured with the attenuated direct beam at 8 (D11) and $3.83 \mathrm{~m}$ (V4). Data reduction was performed with the software Lamp (D11) and BerSANS ${ }^{50}$ (V4). In both cases, the attenuated direct beam was used to obtain absolute intensities.

NSE experiments were performed on the instrument IN15 (ILL) with wavelengths of (longest Fourier time in parentheses) $10 \AA$ ( $58 \mathrm{~ns}), 16 \AA$ ( $206 \mathrm{~ns})$, and $22 \AA$ (598 ns) to cover a $Q$-range from 0.18 to $1.61 / \mathrm{nm}$. Details of the experiments and the methods are explained elsewhere..$^{29,51-53}$

Rheology measurements were performed on an Anton Paar Physica MCR 501 (sodium dodecyl sulfate (SDS)) and with a Malvern Instruments Gemini 200 HR (sodium dodecylbenzenesulfonate(SDBS)), both in cone-plate geometry.

JR 400 (Dow Chemical, USA, see Fig. 1) is a cationically modified hydroxyethylcellulose (cat-HEC) with a molecular weight of about $500000 \mathrm{~g} / \mathrm{mol}$ (polydispersity index (PDI)

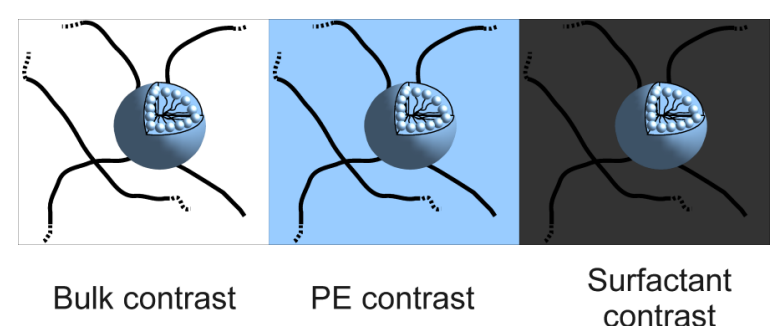

FIG. 2. Different contrasts used in the experiments; in bulk contrast (hydrogenated compounds and deuterated solvent), both PE and surfactant are visible; in pe contrast (hydrogenated $\mathrm{PE}$, deuterated surfactant, and deuterated solvent), only the PE is visible; in surfactant contrast (hydrogenated PE, deuterated surfactant, and $\mathrm{H}_{2} \mathrm{O}: \mathrm{D}_{2} \mathrm{O} 8: 2$ wt:wt as solvent), only the surfactant is visible.

$=1.85,{ }^{19}$ partial molar density in water $1.66 \mathrm{~g} / \mathrm{ml}$ ) and a cationic group on $27 \%$ of the glucose units, resulting in $1000 \mathrm{~g}$ of PE per mol of positive charges. ${ }^{54}$ Its overlap concentration is 0.8 wt. $\%{ }^{47}$ Linear alkylbenzenesulfonate (LAS) (Sasol, Germany) is an anionic surfactant, consisting of a benzenesulfonate headgroup and an alkyl chain with an average length of 12 carbon atoms. It has an average molecular weight of $318 \mathrm{~g} / \mathrm{mol}$, determined by titration with $\mathrm{NaOH}$. Equimolar amounts of $\mathrm{NaOH}$ (97\%, Merck, Germany) were added to obtain sodium dodecylbenzenesulfonate (SDBS).

h-SDS (98.5\%) and d-SDS (99.4\% isotopic purity) were purchased from Sigma-Aldrich and MSD Isotopes, respectively, and were used without further purification.

Surfactant concentrations are given as $Z=$ [polymer charges]/[surfactant charges] or as molar concentrations. The PE concentration was either 0.33 (below overlap concentration) or $1 \mathrm{wt}$ \% (above overlap concentration). Solutions for neutron scattering experiments were prepared in either $\mathrm{D}_{2} \mathrm{O}$ (99.9\% isotopic purity, Euriso-top, France) or a mixture of $\mathrm{H}_{2} \mathrm{O}$ and $\mathrm{D}_{2} \mathrm{O}$ (8:2 wt:wt). Solutions for rheology experiments were prepared in $\mathrm{H}_{2} \mathrm{O}$ from a Millipore System. In the contrast matching experiments, 3 different contrasts were used: Full contrast with h-SDS and $\mathrm{D}_{2} \mathrm{O}$ as solvent where everything is visible, $\mathrm{PE}$ contrast with $\mathrm{d}-\mathrm{SDS}$ and $\mathrm{D}_{2} \mathrm{O}$, where only the $\mathrm{PE}$ is visible, and finally, surfactant contrast with d-SDS and $\mathrm{H}_{2} \mathrm{O}: \mathrm{D}_{2} \mathrm{O}$ (8:2 wt:wt, see Fig. $\mathrm{S1}^{55}$ for the quality of the matching), where only the surfactant is visible (see Fig. 2). Experiments with SDBS have only been performed in full contrast.

\section{RESULTS AND DISCUSSION}

Adding rather small amounts of anionic surfactant to semidilute mixtures of the cationically charged PE JR 400

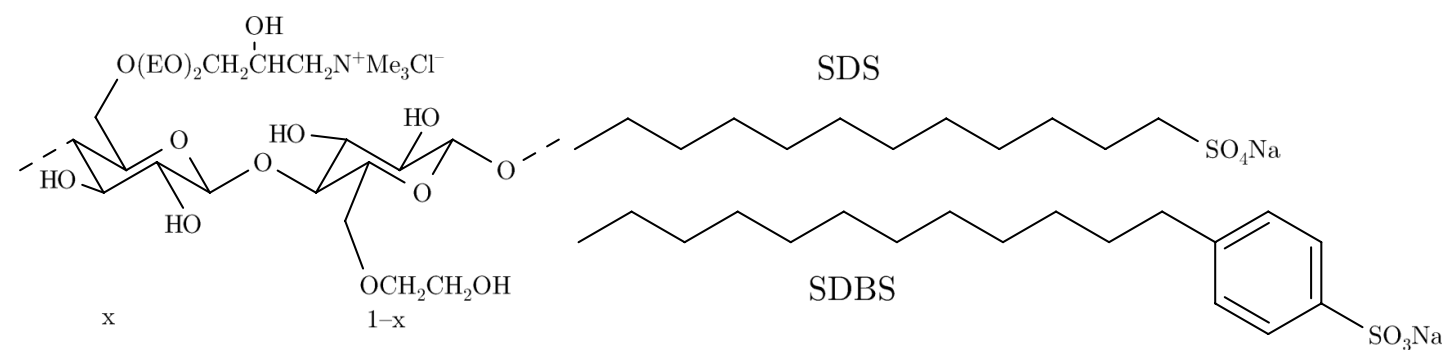

FIG. 1. Chemical structure of JR $400(x=0.27)$ and the surfactants SDS and SDBS. 


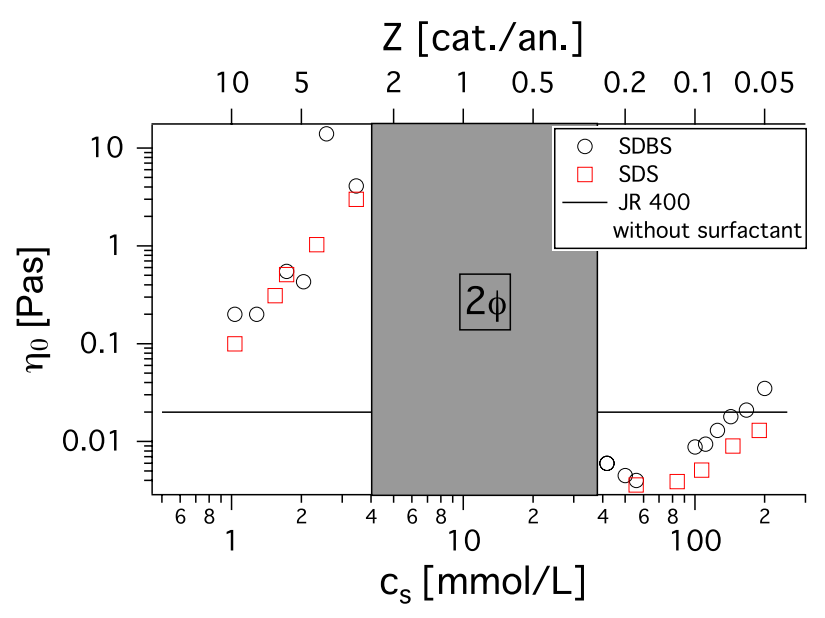

FIG. 3. Zero shear viscosity of mixtures of $1 \mathrm{wt} \%$ JR 400 and surfactant as a function of surfactant concentration; on the PE rich side, the viscosity increases dramatically. On the surfactant rich side, the viscosity is reduced even below the value of the pure PE.

has a quite dramatic influence on the viscosity of the system (see Fig. 3 and Ref. 46). As long as an excess of PE is present, a quite dramatic increase in viscosity can be observed until the system starts to form a solid precipitate around the charge equilibrium. Adding a large excess of surfactant, the precipitate is redissolved and the viscosity drops even below that of the pure PE solution at that concentration. In addition, viscous solutions with an excess of PE near the phase boundary show shear thinning behaviour, ${ }^{47}$ implying that the solution has some structure, which becomes aligned or destroyed when applying sufficient shear.

In this paper, we explore, how different surfactants and surfactant concentrations affect the composition and the length of the rod-like aggregates as much as the freedom of movement of the PE in the aggregates. In addition, the influence of the $\mathrm{PE}$ concentration will be investigated. By doing so, we aim at deriving a self-consistent picture of structure and dynamics in such systems and address the question of the structural origin of the largely increased viscosity.

\section{A. Structure and dynamics of pure polyelectrolyte solution}

Theory ${ }^{56-58}$ predicts an intermediate scattering function with a fast and a slow mode for PEs at high $Q$. However, the fast mode related to the motion of counterions is not in the observable time window for NSE and the intensity stemming from the counterions is negligible anyway. Therefore, only the slow mode is observed at high $Q$. At $Q$ smaller than the polyelectrolyte peak, the 2 modes should not be decoupled and the apparent diffusion coefficient should decrease with increasing $Q$ to reach a plateau. The corresponding diffusion coefficient has been related to the segment length ${ }^{59}$ or the distance between two self-knots. ${ }^{60}$ At even higher $Q$, a linear increase of the apparent diffusion coefficient is expected due to hydrodynamic interactions and internal chain dynamics. Following, e.g., Akcasu, Benmouna, and Han ${ }^{61}$ this increase of the apparent diffusion coefficient persists up to a $Q$ value of about $Q a \approx 1$, where $a$ corresponds to the Kuhn length and a transition to diffusive behaviour is observed.

What is observed for the intermediate scattering function of JR 400 is essentially a monoexponential decay over the investigated $Q$ range (see Fig. $\mathrm{S} 2^{55}$ ). The apparent diffusion coefficient $D_{a p p}$ obtained from fitting a single exponential of the form $S(Q, t)=\exp \left(-D_{a p p} Q^{2} t\right)$ increases linearly with $Q$ (see Fig. S3 ${ }^{55}$ ). Such behaviour was previously also observed in polymer solutions ${ }^{62}$ or microgel particles ${ }^{63}$ and is due to hydrodynamic interactions and internal chain dynamics.

The lacking decrease of the diffusion coefficient at low $Q$ can be understood, when considering that the concentration of the PE is quite low and only a very shallow peak can be observed around $0.41 / \mathrm{nm}$, which is in addition at the low end of the NSE $Q$ range.

Therefore, we can describe the intermediate scattering function as a combination of translational diffusion and hydrodynamic interactions. To account for the latter, Dubois-Violette and de Gennes ${ }^{64}$ derived an approximate form of $S(Q, t)$ for a Zimm chain ${ }^{65}$ which reads

$$
\begin{aligned}
S_{\text {Zimm }}(\Gamma, Q, t)= & \int_{0}^{\infty} \mathrm{d} u \exp \left(-u-(\Gamma t)^{2 / 3} h\left(u /(\Gamma t)^{2 / 3}\right)\right) \\
h(u)= & \frac{2}{\pi} \int_{0}^{\infty} \mathrm{d} x \cos (x u) \\
& \times\left(1-\exp \left(-x^{3 / 2} / \sqrt{2}\right)\right) / x^{2} \\
\Gamma= & \frac{k_{B} T}{6 \pi \eta_{\text {app }}} Q^{3} .
\end{aligned}
$$

The only free parameter in this expression is the apparent solvent viscosity $\eta_{a p p}$. Additionally accounting for translational motion, data have been fitted to

$$
S(Q, t)=\exp \left(-D_{t} Q^{2} t\right)\left((1-A(Q))+A(Q) S_{\text {Zimm }}(\Gamma, Q, t)\right),
$$

where $D_{t}$ is the translational diffusion coefficient and $A(Q)$ is the amplitude of the hydrodynamic contribution. This amounts to 2 global parameters $\left(D_{t}\right.$ and $\left.\Gamma / Q^{3}\right)$ and one additional free parameter $A(Q)$ for every $Q$ value. Even though the JR 400 chain has a relatively long persistence length, the increase of the apparent diffusion coefficient continues beyond the measured $Q$ range. However, while usually an isotropic segment with a length similar to its radius is assumed, here we are dealing with strongly anisotropic segments, and apparently their radius is of greater significance than their length in determining the crossover between the $D_{a p p} \propto Q$ and the $D_{a p p}=$ const . region. Good fit results are obtained (see Fig. $\mathrm{S}^{55}$ ) using Eq. (2) with $A(Q)$ being almost constant within error with a value of about 0.5 (see Fig. S4 ${ }^{55}$ ). The obtained value of $\Gamma / Q^{3}$ is $132 \AA^{3} / \mathrm{ns}$, which corresponds to an apparent viscosity of $1.66 \mathrm{mPa} \mathrm{s}$, which is slightly above the viscosity of $\mathrm{D}_{2} \mathrm{O}$. The diffusion coefficient $D_{t}$ obtained from the fit is $5.1 \AA^{2} / \mathrm{ns}$ corresponding to a length scale of $3.8 \mathrm{~nm}$.

The static scattering of the PE can be described as thin rods with a length of $6.5 \mathrm{~nm}$ and a radius of $0.8 \mathrm{~nm}$ and power law to account for the decrease in intensity with increasing $Q$ at low $Q$ (see Fig. 4). The relatively long persistence length is expected for such an intrinsically stiff $\mathrm{PE}^{20}$ and similar observations have been made for similar compounds. ${ }^{4}$ 


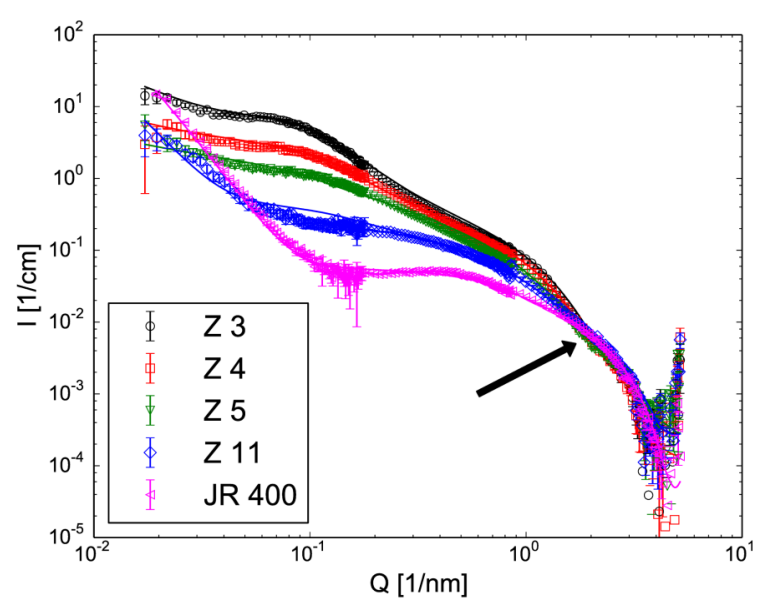

FIG. 4. SANS curves of SDS/JR $4001 \mathrm{wt}$ \%, full contrast, at charge ratios indicated in the graph and $1 \mathrm{wt}$. \% JR 400. The arrow indicates the location of the kink, which is characteristic for the radius of the mixed aggregates.

\section{B. Influence of the surfactant concentration on SDS/JR 400 and SDBS/JR 400 aggregates}

Addition of SDS or SDBS to solutions of $1 \mathrm{wt}$ \% JR 400 causes the formation of rod-like structures, which can be seen in the SANS curves from the $Q^{-1}$ slope at high $Q(>0.51 / \mathrm{nm}$, see Figs. 4 and $\mathrm{S}^{55}$ ). At low $Q$, large clusters which were already observed in the pure PE solution are still present. It has been claimed that their formation is due to counterion entropy. ${ }^{66-68}$ If the amount of surfactant is sufficiently close to the phase boundary, a broad peak can be observed around $0.11 / \mathrm{nm}$. This peak makes it difficult to observe the length of the rod like aggregates.

We performed measurements with different contrasts to resolve the internal structure of the aggregates as different features are seen in different contrasts (see Fig. 2). The rodlike aggregates are similarly observed in all contrasts (see Figs. S6-S $11^{55}$ ). This means that they have a relatively homogeneous structure along their long axis and that they consist of both PE and surfactant. The kink at about $21 / \mathrm{nm}$ indicated by the arrow in Fig. 4, which is the characteristic of the radius of the rods, is situated at slightly higher $Q$ in surfactant contrast (see Figs. S8 and $\mathrm{S}^{55}$ ). This indicates that the radial structure is not entirely homogeneous and the PE is preferentially located at the outer part of the rods.

To describe the high $Q$ part of the SANS curves in detail, we chose to refine our model of homogeneous rods from previous publications. ${ }^{46,47,49}$ In contrast to those, here we describe the cross-sectional structure of the rods with a coreshell model. The rods have an outer shell of outer radius $R_{0}$ and inner radius $R_{1}$, which consists purely of polyelectrolyte. Below the outer shell, a mixed layer of outer radius $R_{1}$ and inner radius $R_{2}$ consisting of both polyelectrolyte and surfactant is present. The core has an outer radius $R_{2}$ and consists purely of surfactant (see Fig. 5). The polyelectrolyte is either free or incorporated in the mixed aggregates, so that the scattering intensity at high $Q$ is given by

$$
I_{h Q}=\frac{\phi_{\text {surf }}+x_{p a} \phi_{p e}}{V_{a g g}} P_{a g g}+\frac{\left(1-x_{p a}\right) \phi_{p e}}{V_{p e}} P_{p e},
$$

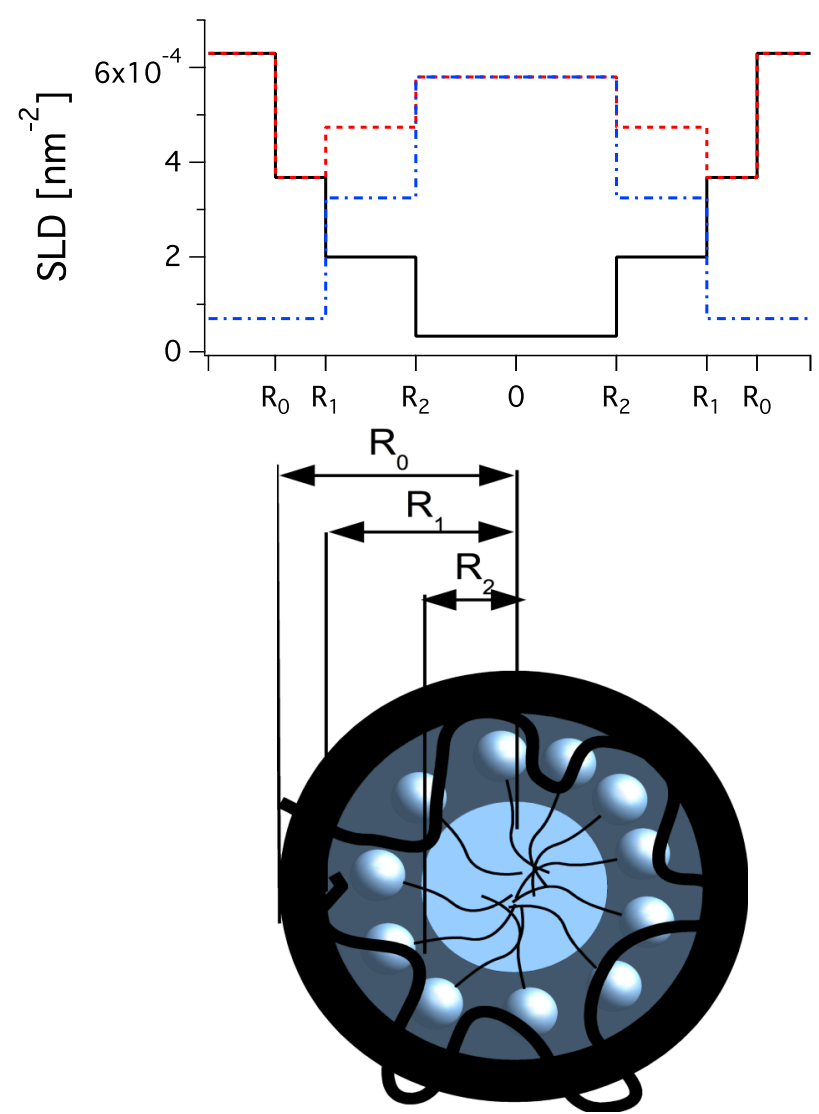

FIG. 5. The cross section of the rod-like aggregates is described as a core consisting of only surfactant, a mixed layer consisting of PE and surfactant, and an outer shell consisting of only PE. The resulting scattering length density profile is shown on top for different contrasts; black line: full contrast, dashed red line: PE contrast, dashed-dotted blue line: surfactant contrast, and the contrast of the mixed layer shown here assumes that it consists of equal amounts of PE and surfactant.

where $\phi_{\text {surf }}$ and $\phi_{p e}$ are the volume fraction of the surfactant and the polyelectrolyte, respectively, and $x_{p a}$ is the fraction of the polyelectrolyte volume fraction which is incorporated in the aggregates. $V_{a g g}$ and $V_{p e}$ are the volume of the mixed aggregates and the small rods, which describe the scattering of the pure polyelectrolyte and $P_{a g g}$ and $P_{p e}$ are their form factors (see Eq. (4)), where the latter describes the chain scattering. For both $P_{a g g}$ and $P_{p e}$, the form factor for randomly oriented core-shell rods with $n$ shells in radial direction $\left(P_{\text {agg }}: 2\right.$ shells, $P_{p e}: 0$ shells) is used,

$P\left(Q, R_{0, \ldots, n}, \Delta S L D_{0, \ldots, n}, L\right)=\left(\sum_{i=0}^{n} F\left(Q, R_{i}, \Delta S L D_{i}, L\right)\right)^{2}$,

with the radii of the shells $R_{i}$ and the corresponding scattering length density differences $\triangle S L D_{i}$, where $\triangle S L D_{i}=S L D(R$ $\left.<R_{i}\right)-S L D\left(R>R_{i}\right)$. The thickness of a shell $i$ is given by $R_{i}-R_{i+1}$, where $R_{0}$ is the outer radius and $R_{n}$ is the radius of the core. The scattering amplitude of a rod is given by

$F(Q, R, \triangle S L D, L)$

$$
=\pi R^{2} L \Delta S L D \int_{0}^{1} \frac{4 J_{1}\left(Q, R \sqrt{1-x^{2}}\right) \sin (Q L x / 2)}{Q^{2} R \sqrt{1-x^{2}} L x} \mathrm{~d} x,
$$


where $J_{1}$ is the first order Bessel function of the first kind and the scattering intensity is given by $I={ }^{1} N P \cdot S+I_{\text {inc }}$, where $I_{i n c}$ is the incoherent scattering intensity, $S$ is the interparticle structure factor, and ${ }^{1} N$ is the particle number density. For monodisperse particles, it is related to the volume fraction $\phi$ by ${ }^{1} N=\phi / V_{p}$, where $V_{p}$ is the volume of the particle (for the polydisperse case, the number average of $V_{p}$ ). Equation (3) assumes that all of the surfactant is in the aggregates, which is a valid assumption, as the critical aggregation concentration (the surfactant concentration at which the surfactant starts adsorbing on the PE) is known to be several orders of magnitude smaller than the critical micelle concentration $(\mathrm{cmc}){ }^{19}$ The form factor of the PE in its native state is assumed to remain unchanged, in order to keep the number of free parameters small.

Assuming that the aggregates are homogeneous along their long axis, the fraction of PE and surfactant in the mixed middle shell of the aggregates is related to the radii of the inner shells $R_{1}$ and $R_{2}$ via the circular cross sectional area. Given a circle of radius $R_{0}$ and a shell with outer radius $R_{1}$ and inner radius $R_{2}$, where $R_{0}>R_{1}>R_{2}$ (see Fig. 5), the fraction of the area of the circle covered by the shell is given by $x=\frac{R_{1}^{2}-R_{2}^{2}}{R_{0}^{2}}$. In the mixed aggregates, the fraction of the area (and, as we assume a homogeneous structure, also the volume) is determined by the fraction of surfactant $\left(x_{m i x, s}\right)$ and polyelectrolyte $\left(x_{m i x, p e}\right)$ in the mixed shell, and the rest of the polyelectrolyte in the aggregates, $1-x_{\text {mix,pe }}$, is in the outer shell. The radius of the surfactant core $R_{2}$ is given by

$$
R_{2}=\sqrt{1-x_{m i x}, s} R_{0},
$$

and the radius of the mixed shell is given by

$$
R_{1}=\sqrt{\left(x_{m i x, s}+x_{m i x, p e}\right) R_{0}^{2}+R_{2}^{2}} .
$$

Note that $x_{m i x, p e}$ is the fraction of PE in the mixed shell of the polyelectrolyte in the aggregates, so that $\phi_{m i x, p e}=x_{p a} x_{m i x, p e}$ $\phi_{p e}$. This model was chosen over a simple homogeneous rod $\left(x_{m i x, s}=1\right.$ and $\left.x_{m i x, p e}=1\right)$ or a core-shell structure without a mixed middle layer $\left(x_{m i x, s}=0\right.$ and $\left.x_{m i x, p e}=0\right)$ as it turned out that the mixed shell was necessary to always obtain a good description with the same set of parameters for all contrasts at a given surfactant concentration. While $R_{0}$ and $x_{p a}$ can be deduced from measurements in full or PE contrast, $x_{m i x, s}$ and $x_{m i x, p e}$ have almost no effect in these measurements. Their effect only becomes visible in surfactant contrast (see Fig. 6). This also explains why we were able to describe both the data from full and PE contrast with the same set of parameters but needed to assume a different radius for the description of the data with matched PE in our previous publication. ${ }^{49}$

Fitting Eq. (3) to the data with $x_{p a}, x_{m i x, s}$, and $x_{m i x, p e}$ and $R_{0}$ as free parameters (where the same set of parameters is used for all contrasts) yields relatively constant and robust values of about $2 \mathrm{~nm}$ for the outer radius of the aggregates (see Fig. 7), where aggregates with SDBS seem to yield slightly larger radii. Measurements in surfactant contrast suffer from a relatively low overall contrast and a high incoherent background, due to the high fraction of $\mathrm{H}_{2} \mathrm{O}$ in the solvent in addition to the generally low scattering intensity owing to the low surfactant concentrations (less than $3.5 \mathrm{mM}$ ) in the samples. Therefore,

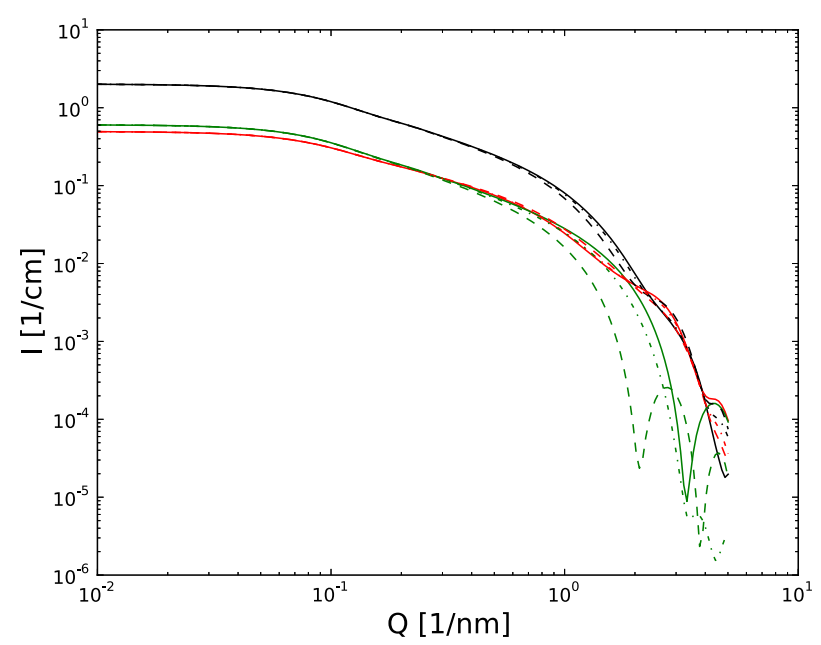

FIG. 6. Different contrasts with different values for $x_{m i x, s}$ and $x_{m i x, p e}$ : black: full contrast, red: PE contrast, green: surfactant contrast. Full lines: $x_{m i x, s}$, $x_{\text {mix }, p e}=0$; dashed-doted lines: $x_{\text {mix }, s}, x_{\text {mix }, p e}=0.5$; dashed lines: $x_{\text {mix }, s}$, $x_{\text {mix }, p e}=1$. While the distribution of the material in the aggregates has only little effect on the scattering curve in full and PE contrast, it can be easily seen in surfactant contrast. Other parameters as for $1 \mathrm{wt}$ \% JR 400, Z $=3$ (SDS), see Table S I, ${ }^{55} S_{l o r}=1, A_{p l}=0$.

only for the 2 highest SDS concentrations, SANS curves have been recorded in that contrast. It can be seen that the mixed layer accounts for the most part of the aggregates and only a relatively thin outer layer of pure PE and surfactant core remains. Another interesting observation, especially in the light of recent findings concerning the importance of hydrophobic interaction for the solubility of cellulose by Lindman, Karlström, and Stigsson ${ }^{69}$ and Medronho et al.,${ }^{70}$ is the vanishingly small surfactant core $R_{2}$ (Fig. 7). This means that the water soluble PE can be found throughout the hydrophobic surfactant core. Merta et al. $^{71}$ investigated complexes from pDADMAC and sodium palmitate. They also observed rod-like particles and using deuterated sodium palmitate, they found a core-shell structure with an outer PE shell and a surfactant core. However,

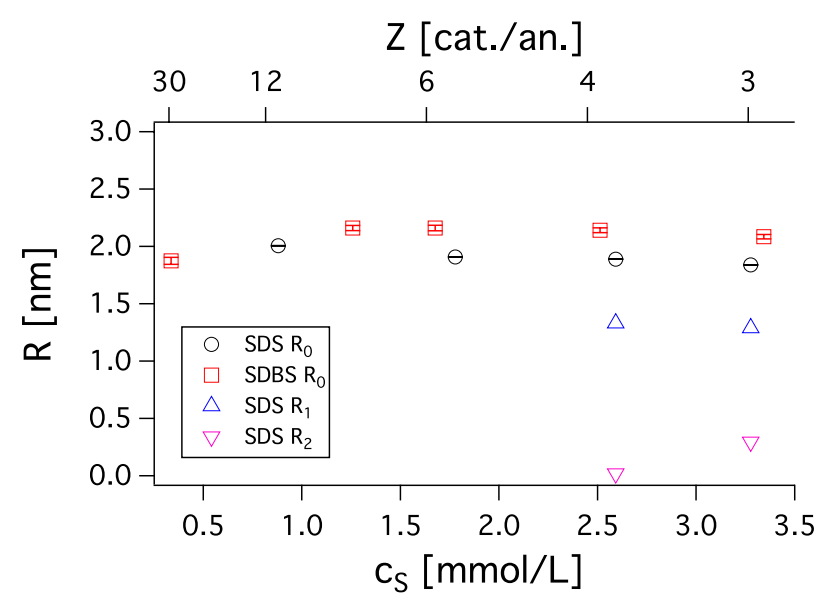

FIG. 7. Radii of aggregates as a function of surfactant concentration (bottom) and charge ratio (top) for SDS and SDBS at a JR 400 concentration of 1 wt. \%; the radius is relatively constant and SDBS aggregates are slightly larger. The inner radii $R_{1}$ and $R_{2}$ are only shown for the highest surfactant concentrations, where measurements in surfactant contrast were performed. The PE penetrates relatively deeply into the aggregate and leaves only a vanishingly small surfactant core and a small corona with only PE. 


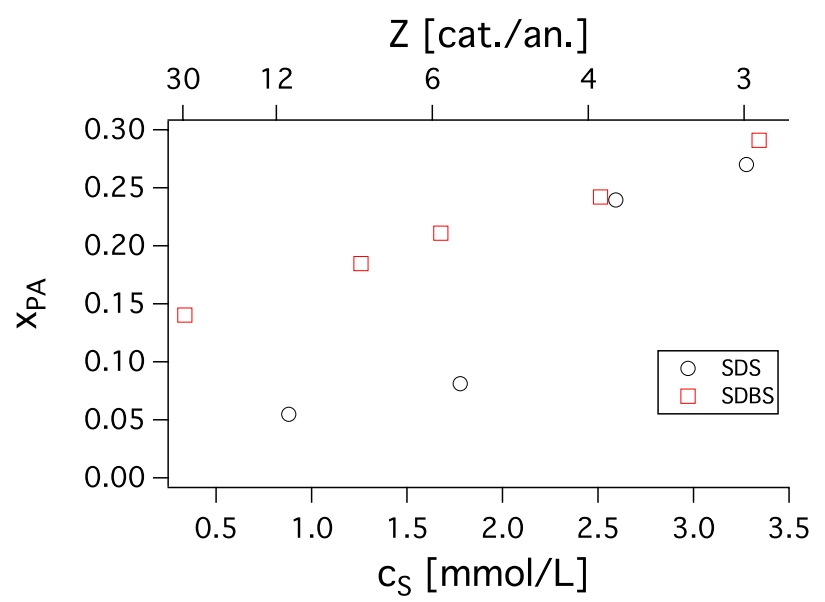

FIG. 8. Fraction of PE in aggregates $x_{p a}$ as a function of surfactant concentration (bottom) and charge ratio (top) for SDS and SDBS; at lower surfactant concentrations, SDBS is generally more efficient in incorporating PE in the aggregates.

the core radii were somewhat small for the surfactant used. It is quite possible that they observed a similar structure as found here, where the PE penetrates deeply into the surfactant core, which results in values a bit too small for the surfactant core, when described with a model with only a single shell.

The fraction of bound PE $x_{p a}$ increases as the surfactant concentration increases for both SDS and SDBS (see Fig. 8). However, at low concentrations, SDBS seems to be significantly more efficient in binding PE in the aggregates. This can be understood when keeping in mind that the head group of SDBS is more hydrophobic than that of SDS, and SDBS forms rod-like micelles more easily, upon adding salt. For example, $200 \mathrm{mM} \mathrm{NaCl}$ at $25^{\circ} \mathrm{C}$ is sufficient to significantly increase the size of SDBS micelles at $70 \mathrm{mM}$ surfactant while even $300 \mathrm{mM}$ of $\mathrm{NaCl}$ is insufficient to achieve the same effect with SDS micelles at the same concentration and temperature (see, e.g., Table I of Mazer, Benedek, and Carey ${ }^{72}$ and Table II of Cheng and Gulari ${ }^{73}$ ). An interesting detail is revealed, when converting $x_{p a}$ to the ratio of charges in the aggregates $\left(c_{p a}\right.$ $=\phi_{p e} x_{p a} \rho_{J R 400} / M_{J R 400}$, with $\rho_{J R 400}=1.66 \mathrm{~g} / \mathrm{ml}$ and $M_{J R 400}$ $=1000 \mathrm{~g} / \mathrm{mol}) c_{p a} / c_{s}$. At low surfactant concentrations, aggregates with SDBS have an excess of positive PE charges, while SDS aggregates have an excess of surfactant charges. As more surfactant is added, the aggregates are almost neutral before they precipitate at $Z<3$ (see Fig. 9).

At low $Q$, an additional increase in intensity with decreasing $Q$ is observed, which is due to the formation of large, loosely bound PE clusters. ${ }^{66-68,74}$ This increase in intensity can be described with a power law with an exponential cutoff. If the surfactant concentration is sufficient, a broad peak can be observed, which makes it impossible to determine the length of the aggregates. In our empirical approach, this correlation peak is described by a Lorentzian structure factor $S_{l o r}$. The resulting scattering intensity is given by

$$
I=I_{h Q} S_{l o r}+A_{p l} Q^{-f} \exp \left(-Q / Q_{c u t}\right),
$$

where $A_{p l}$ is the amplitude of the power law, $f$ is its exponent and $Q_{c u t}$ determines the cutoff of the power law, and $I_{h Q}$ is

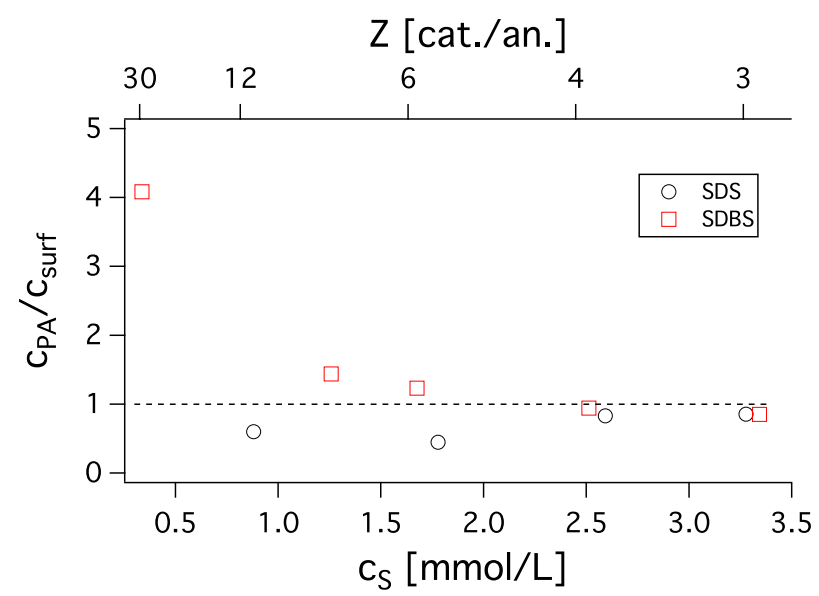

FIG. 9. Ratio of PE charge concentration to surfactant concentration in aggregates with 1 wt. \% JR 400 as a function of surfactant concentration (bottom) and charge ratio (top) for SDS and SDBS, and the dashed line indicates charge equilibrium; while aggregates with SDBS have an excess of PE charges, aggregates with SDS have an excess of surfactant charges. Close to the phase boundary, the aggregates are almost uncharged in both cases.

given by Eq. (3). The Lorentzian peak is given by

$$
S_{l o r}=\frac{A_{l o r} \sigma}{\left(Q-2 \pi / R_{l o r}\right)^{2}+\sigma^{2}}+1
$$

where $A_{l o r}$ determines the peak height, $\sigma$ determines its width, and $R_{l o r}$ determines the peak position (and is given by the concentration of rods: $R_{\text {lor }}={ }^{1} N^{-1 / 3}$, where ${ }^{1} N$ is the particle number density of the rods). However, the length of the aggregates needs to be known to disentangle the contribution from the large low $Q$ clusters and the smaller rod-like aggregates. For somewhat higher surfactant concentrations $(Z<6)$, it is not clear from the SANS measurements if the rod-like aggregates are very long and the slope at very low $Q$ is due to their form factor or if they actually reach a plateau near the position of the peak (see, e.g., Fig. 4).

Therefore, NSE measurements were performed to investigate the dynamics of the systems, which also depends strongly on the length. In the $Q$ region where the static scattering is dominated by the rod-like aggregates, the apparent diffusion coefficients, which are obtained by fitting an expression of the form $S(Q, t)=\exp \left(-D_{a p p} Q^{2} t\right)$ to the intermediate scattering function, are relatively constant, which means they show diffusive behavior and it is reasonable to assume that the observed effect is the diffusion of the rod-like aggregates. At smaller $Q$ or when using longer wavelengths, which allow to access longer Fourier times, a smaller $D_{a p p}$ is observed, because the relaxation of the large aggregates becomes visible. At higher $Q$, when the static scattering is dominated by the PE chains, $D_{a p p}$ increases. See Fig. $\mathrm{S}^{55}$ for an example.

The diffusion of a rod can be described with the Broersma equation ${ }^{75}$ for the translational $\left(D_{\text {rod }}\right)$ and the rotational $\left(\Theta_{\text {rod }}\right)$ diffusion coefficient,

$$
\begin{aligned}
D_{\text {rod }} & =\left(\frac{k_{b} T}{3 \pi \eta L}\right)\left(\delta-0.5\left(\gamma_{\|}(\delta)+\gamma_{\perp}(\delta)\right)\right), \\
\Theta_{\text {rod }} & =\left(\frac{3 k_{B} T}{\pi \eta L^{3}}\right)(\delta-\zeta(\delta)),
\end{aligned}
$$


where $\delta=\ln (L / R), \gamma_{\|}=1.27-7.4(1 / \delta-0.34)^{2}, \gamma_{\perp}=0.19$ $-4.2(1 / \delta-0.39)^{2}$ and $\zeta=1.45-7.2(1 / \delta-0.27)^{2}$. This results in an intermediate scattering function with 2 decays,

$$
\begin{aligned}
S_{\text {rod }}(Q, t)= & s_{0}(Q) \exp \left(-D_{\text {rod }} Q^{2}\right) \\
& +s_{1}(Q) \exp \left(-\left(D_{\text {rod }} Q^{2}+6 \Theta_{\text {rod }}\right) t\right),
\end{aligned}
$$

with the amplitudes

$$
\begin{aligned}
& s_{0}(Q)=\left(\frac{2}{Q L} \int_{0}^{\frac{Q L}{2}} J_{0}(z) \mathrm{d} z\right)^{2}, \\
& s_{1}(Q)=5\left(\frac{1}{Q L}\left(-3 J_{1}\left(\frac{Q L}{2}\right)+\int_{0}^{\frac{Q L}{2}} J_{0}(z) \mathrm{d} z\right)\right)^{2},
\end{aligned}
$$

where $J_{n}$ is the $n$th order spherical Bessel function. For the sake of computational simplicity, the diffusion of the PE is described with its linearly $Q$-dependent apparent diffusion coefficient and the unnormalized intermediate scattering function reads

$$
\begin{aligned}
S(Q, t)= & \frac{\phi_{\text {surf }}+x_{p a} \phi_{p e}}{V_{a g g}} P_{a g g} \cdot S_{\text {rod }}\left(Q, t, R_{0}, L\right) \\
& +\frac{\left(1-x_{p a}\right) \phi_{p e}}{V_{p e}} P_{p e} \cdot \exp \left(-D_{a p p, p o l}(Q) Q^{2} t\right),
\end{aligned}
$$

where $P_{a g g}$ is the form factor of the rod-like aggregates and $P_{p e}$ is the form factor of the chains. With all parameters except the length of the aggregates $L$ known from SANS, it is now possible to obtain the length from fitting the NSE data using Eq. (13). Fitting the different $Q$ values separately yields relatively constant values for $L$ for $Q$ values above $0.41 / \mathrm{nm}$ for both SDS and SDBS (see Figs. 10 and S12-S15 ${ }^{55}$ ) when using a wavelength of $10 \AA$, which does not reach Fourier times long enough for the relaxation of the large clusters to have an effect on $S(Q, t)$. Therefore, the $10 \AA$ data were fitted simultaneously using only a single $L$ for all curves (see Figs. 11 and $\mathrm{S} 16-\mathrm{S} 20^{55}$ ) and the 16 and $22 \AA$ measurements have been omitted as they are influenced by the relaxation of large clusters. The results are summarized in Fig. 12. The lengths

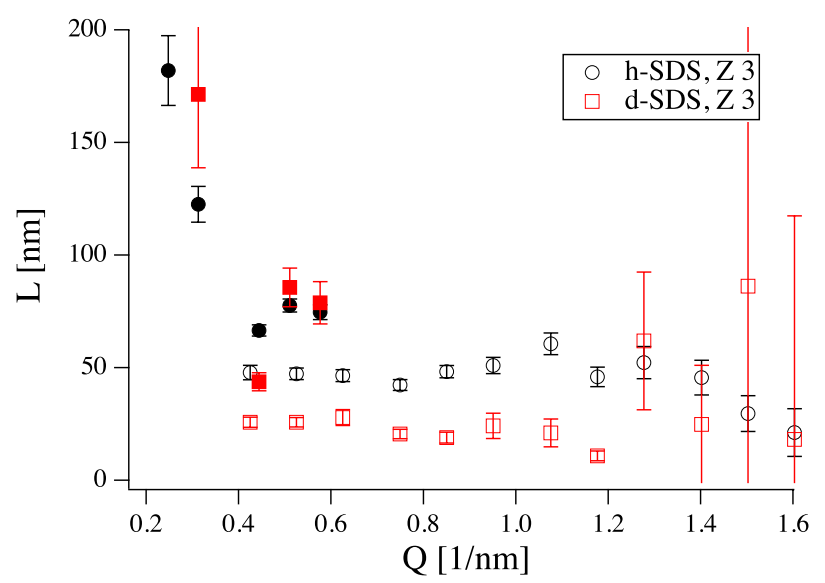

FIG. 10. Lengths of aggregates obtained from fits as a function of $Q$, for SDS/JR 400, $1 \mathrm{wt} . \%$ at the charge ratio indicated in the graph; open symbols: $10 \AA$, closed symbols: 16 or $22 \AA$ wavelength; at low $Q$ and using longer wavelengths, which allows to reach longer Fourier times, the lengths obtained from the fits are starting to increase due to the influence of larger clusters. The lengths obtained from samples with deuterated surfactant are significantly shorter, which is due to the mobility of the PE within the aggregates.

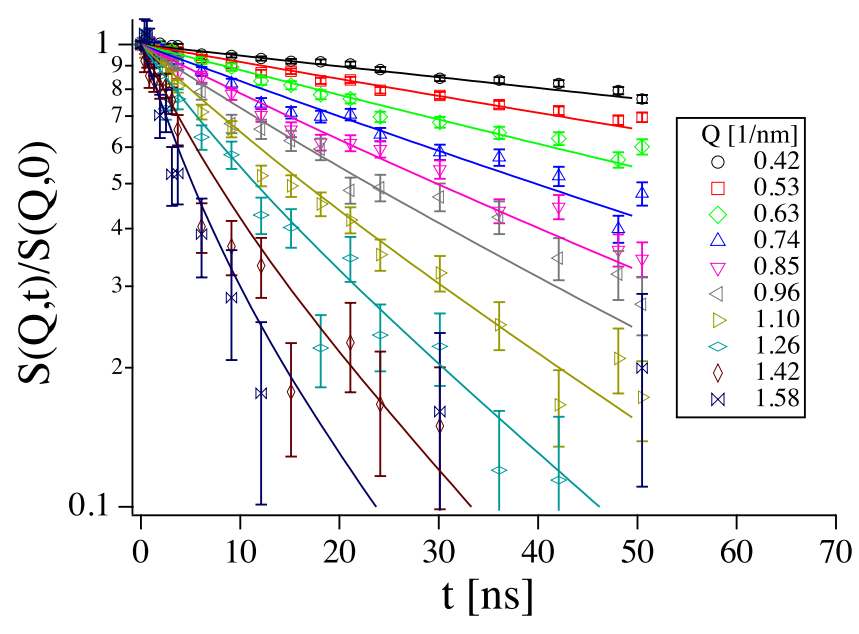

FIG. 11. Intermediate scattering function of SDBS/JR $4001 \mathrm{wt} \%, Z=3$; lines are fits to Eq. (13) with $L$ as the only free parameter.

obtained for different concentrations are relatively constant, and only at the lowest surfactant concentration, they seem to become a little shorter and SDS aggregates appear to be somewhat longer than SDBS aggregates. As the structure peak is less pronounced for SDBS/JR 400 aggregates, it is possible to determine the length with SANS at sufficiently low SDBS concentrations and the agreement with the results from NSE at higher SDBS concentrations is remarkably good (see Fig. 12). It is somewhat surprising that the rod-like SDS aggregates are longer than the SDBS aggregates, despite the latter forming rod-like micelles upon addition of less salt. ${ }^{72,73}$ However, while the model of homogeneous rods fits the SDBS data almost perfectly (see Fig. $5^{55}$ ), some slight deviations can be seen in the SDS data, which hint towards some slight heterogeneities along the rod-like aggregates (see Fig. 4). So, while SDS forms longer rods, SDBS forms more homogeneous rods.

It could be expected that NSE data of the same samples in PE contrast could be described using the same parameters, only adapting the contrasts. However, looking at Figs. 10 and $\mathrm{S} 12-\mathrm{S} 14,{ }^{55}$ it is found that the lengths obtained with d-SDS

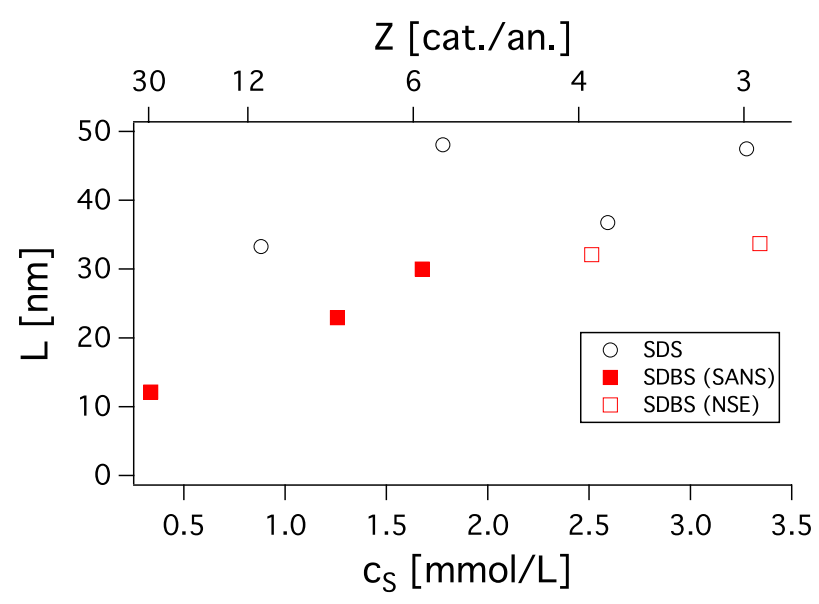

FIG. 12. Lengths of aggregates obtained from fits as a function of surfactant concentration (bottom) and charge ratio (top) for SDS (all from NSE) and SDBS at a JR 400 concentration of 1 wt. \%; the length of the aggregates is relatively constant with concentration, and SDBS aggregates are slightly shorter than SDS aggregates. 
are systematically shorter, than those obtained with h-SDS, which means that the relaxation is faster than it should be. This can be attributed to the PE in the aggregates not being tightly bound and retaining part of its freedom of movement. Therefore, NSE allows to obtain additional information on the internal structure of the aggregates and to quantify the fraction of the PE which can still move in the aggregates; $S_{\text {rod }}$ in Eq. (13) has been replaced by

$$
\begin{aligned}
S_{\text {rod,free }}(Q, t)= & S_{\text {rod }}(Q, t)\left(\left(1-a_{\text {free }}\right)\right. \\
& \left.+a_{\text {free }} \exp \left(-D_{\text {app }, p o l}(Q) Q^{2} t\right)\right),
\end{aligned}
$$

where $a_{\text {free }}$ is a measure for the fraction of freely moving PE in the aggregates and we assume that the dynamics of the "free" $\mathrm{PE}$ in the aggregates is the same as for the completely unbound PE. Therefore, $D_{a p p}$ obtained from the linear fit to the data from the pure PE (see Fig. S3 ${ }^{55}$ ) was used. The NSE data with dSDS have been fitted with the length obtained from the samples in full contrast with $a_{\text {free }}$ as the only free parameter (see Figs. $\mathrm{S} 21-\mathrm{S} 24^{55}$ ) and the results are summarized in Fig. S25. ${ }^{55}$ The parameter seems to increase slightly with decreasing surfactant concentration, which means that the PE is more tightly bound at higher surfactant concentrations. It is tempting to compare $a_{\text {free }}$ to the fraction of $\mathrm{PE}$ in the mixed layer $x_{\text {mix,pe }}$ but the fraction of PE in the outer shell is significantly larger, which means that here one is not really seeing the same.

Knowing the lengths of the aggregates, we proceeded to fit the remaining parameters of Eq. (8) to the SANS curves, to obtain an expression which describes the whole $Q$-range. $R_{l o r}$ was calculated from the known particle number density of the aggregates ${ }^{1} N$,

$$
\begin{gathered}
{ }^{1} N=\frac{\phi_{\text {surf }}+x_{p a} \phi_{p i}}{V_{\text {agg }}}, \\
R_{\text {lor }}={ }^{1} N^{-1 / 3} .
\end{gathered}
$$

The fact that this approach describes the peak position relatively well supports the hypothesis that the peak does stem from correlations between different rodlike aggregates. The fractal dimension $f$ of the low-Q increase in intensity appears to decrease upon the addition of surfactant (see Tables S I-S III ${ }^{55}$ ). This might indicate that the large clusters adapt a

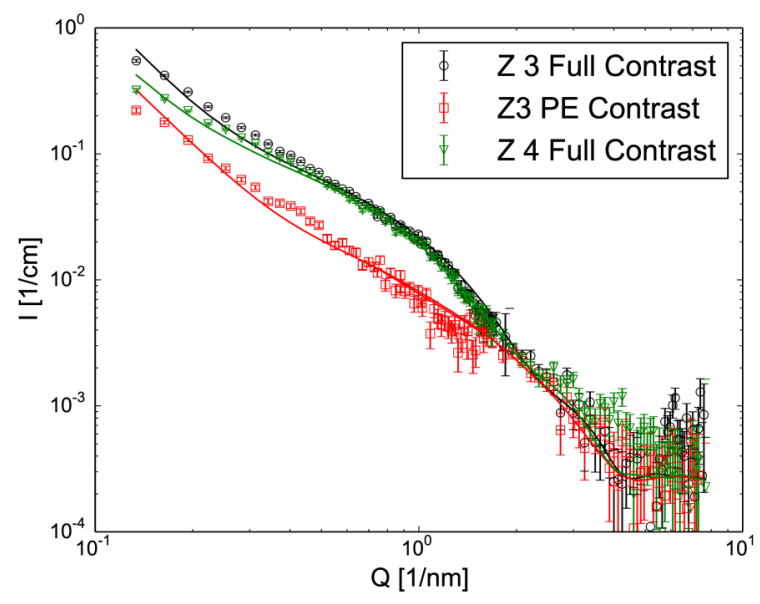

FIG. 13. SANS curves of SDS/JR 4000.33 wt. $\%$ at charge ratios and contrasts indicated in the graph.

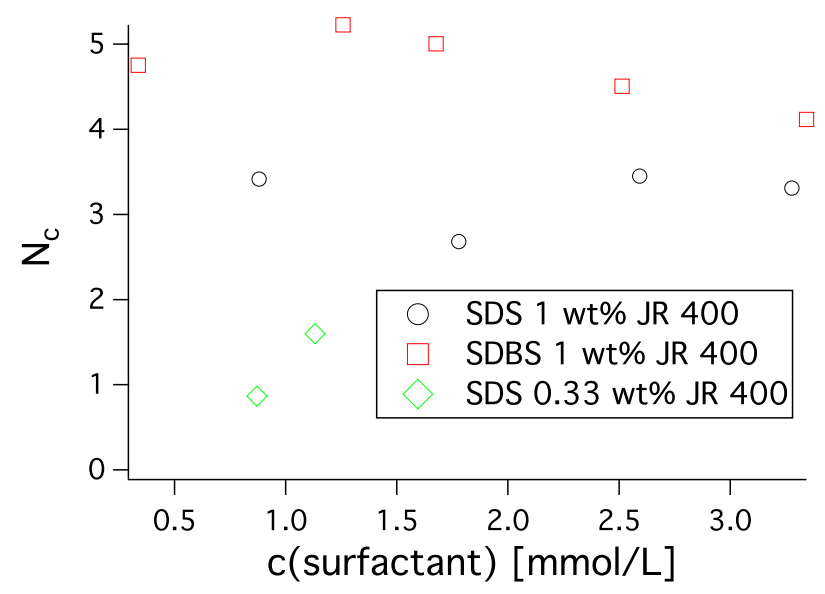

FIG. 14. Number of PE chains per cross sectional area of the aggregates for the surfactants and PE concentrations indicated in the graph calculated according to Eq. (17).

somewhat more stretched conformation. However, the values differ from contrast to contrast and the exact value may depend on experimental parameters such as the shear history of the sample which have not been controlled.

\section{Influence of PE concentration}

To gain a better understanding of the structural reasons for the highly enhanced viscosity, we performed additional SANS measurements at a PE concentration of $0.33 \mathrm{wt}$. \% which is below the overlap concentration and charge ratios of 3 and 4, where no pronounced effect on viscosity can be observed. The SANS curves look qualitatively similar, with rod-like aggregates forming (see Fig. 13). However, fitting the data shows that $x_{p a}$ is related to the overall surfactant concentration rather than the charge ratio (see Figs. S26 and S27 $7^{55}$ ) and at lower PE concentrations, the surfactant is significantly less efficient in binding $\mathrm{PE}$ in aggregates even close to the phase boundary, which is a bit below $Z=3$ for these low PE concentrations as well. Therefore, the amount of PE bound in the aggregates does not only decrease linearly with PE concentration at a given charge ratio but rather quadratically, if we assume that $x_{p a}$ scales roughly linearly with the surfactant concentration. The number of PE chains per cross sectional area of the aggregates calculated as

$$
N_{c}=\frac{\phi_{p a} /\left(\phi_{p a}+\phi_{s}\right)}{R_{p e}^{2} / R_{0}^{2}}
$$

is shown in Fig. 14. So, for a strongly increased viscosity, it is not only necessary that enough aggregates are formed but that they also need to have the right composition.

\section{Summary}

We have investigated aqueous complex forming mixtures of the cationic, cellulose based PE JR 400 with the anionic surfactants SDS and SDBS at different concentrations with SANS and NSE. Such solutions show a remarkable increase in viscosity at sufficiently high PE concentration (>0.5 wt. \%) and upon approaching the phase boundary induced by surfac- 
tant addition. To gain deeper insight into the mesoscopic structure, we were comparing 3 different contrasts for the SDS/JR 400 system. In full contrast both PE and surfactant are visible. In PE contrast only the polyelectrolyte is visible and in surfactant contrast, the scattering length of the PE is matched, so that only the surfactant is visible. This was done by using deuterated SDS instead of hydrogenated SDS and by changing the isotopic composition of the water.

SANS measurements show the formation of mixed rodlike aggregates, which interconnect several PE chains, thereby enhancing the viscosity. At a PE concentration of $1 \mathrm{wt} \%$, both SDS and SDBS are able to significantly enhance the viscosity, where viscosities with SDBS containing samples are somewhat higher. This is reflected in the SANS data, as it is seen that SDBS is slightly more efficient in binding the PE in aggregates and the fraction of $\mathrm{PE}$ in the aggregates increases with surfactant concentration. Contrast matching shows that the PE is preferentially located on the outside of the aggregates but penetrates deeply into the surfactant core of the aggregates.

An interaction peak and large scale clusters render it very complicated to discern the length of the aggregates from the SANS data in most cases. Therefore, NSE measurements were performed, from which the length of the rods could be determined using the Broersma equation. ${ }^{75}$ The rods are on the order of $40 \mathrm{~nm}$ in length, with little changes with surfactant concentration. Additional measurements with deuterated surfactant made it possible to show that the PE in the aggregates is still free to move to some extent, where this is more pronounced at lower surfactant concentrations.

Measurements at lower PE concentration but the same ratio between $\mathrm{PE}$ and surfactant demonstrate that the fraction of PE incorporated in the aggregates depends on the surfactant concentration rather than the ratio between PE and surfactant, which means that the amount of PE in aggregates drops quite rapidly with concentration. Therefore, in addition to having less aggregates in the solution, the aggregates are not able to interconnect several PE chains and hence are not able to increase the viscosity (see Fig. 15).

In summary, by combining SANS and NSE, we have gained a significantly improved understanding of the mesoscopic origins of viscosity enhancement in these oppositely charged PE/surfactant complexes. This detailed structural picture is complemented by the finding that the PE chains in such complexes still have a remarkably high degree of mobility, despite the largely enhanced viscosity. The improved quantita-
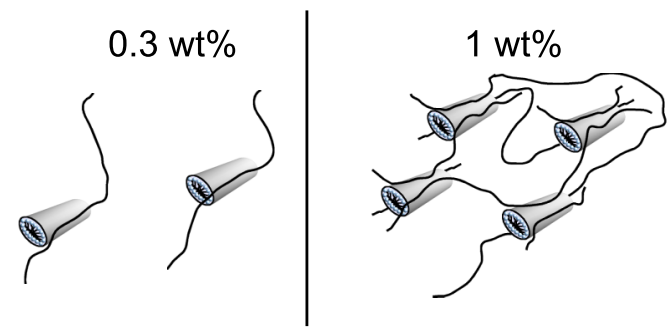

FIG. 15. At low PE concentrations, the fraction of PE in the aggregates is too low to form a significant amount of cross-links between different PE chains (left), whereas at higher concentrations, the aggregates act as cross-links as they connect several PE chains. tive understanding of such PE/surfactant complexes then will be instrumental for developing their applications in various fields of formulations forward.

\section{ACKNOWLEDGMENTS}

Financial support from the BMBF Project No. 05K13KT1 is gratefully acknowledged as well as allocation of beamtime by the ILL and HZB.

${ }^{1}$ J. Skolnick and M. Fixman, "Electrostatic persistence length of a wormlike polyelectrolyte," Macromolecules 10, 944-948 (1977).

${ }^{2}$ T. Odijk, "Polyelectrolytes near the rod limit," J. Polym. Sci., Polym. Phys. Ed. 15, 477-483 (1977).

${ }^{3}$ F. Bonnet, R. Schweins, F. Boué, and E. Buhler, "Suppression of aggregation in natural-semiflexible/flexible polyanion mixtures, and direct check of the OSF model using SANS,' Europhys. Lett. 83, 48002 (2008).

${ }^{4} \mathrm{E}$. Josef and H. Bianco-Peled, "Conformation of a natural polyelectrolyte in semidilute solutions with no added salt," Soft Matter 8, 9156-9165 (2012).

${ }^{5}$ E. D. Goddard and R. B. Hannan, "Cationic polymer/anionic surfactant interactions,” J. Colloid Interface Sci. 55, 73-79 (1976).

${ }^{6} \mathrm{~L}$. Piculell and B. Lindman, "Association and segregation in aqueous polymer/polymer, polymer surfactant, and surfactant surfactant mixtures - similarities and differences," Adv. Colloid Interface Sci. 41, 149-178 (1992).

${ }^{7}$ E. D. Goddard, "Polymer/surfactant interaction-its relevance to detergent systems," J. Am. Oil Chem. Soc. 71, 1-16 (1994).

${ }^{8}$ S. Kosmella, J. Kötz, K. Shirahama, and J. Liu, "Cooperative nature of complex formation in mixed polyelectrolyte-surfactant systems," J. Phys. Chem. B 102, 6459-6464 (1998).

${ }^{9}$ E. F. Marques, O. Regev, A. Khan, M. D. G. Miguel, and B. Lindman, "Interactions between catanionic vesicles and oppositely charged polyelectrolytes phase behavior and phase structures," Macromolecules 32, 6626-6637 (1999).

${ }^{10}$ Y. Yamaguchi, Y. Inaba, H. Uchiyama, and H. Kunieda, "Anomalous phase behavior of water-soluble polyelectrolyte and oppositely charged surfactants," Colloid Polym. Sci. 277, 1117-1124 (1999).

${ }^{11}$ A. Svensson, J. Sjöström, T. Scheel, and L. Piculell, "Phases and structures of a polyion-surfactant ion complex salt in aqueous mixtures: Cationic hydroxyethyl cellulose with dodecylsulfate counterions," Colloids Surf., A 228, 91-106 (2003).

${ }^{12}$ S. Zhou, C. Xu, J. Wang, P. Golas, J. Batteas, and L. Kreeger, "Phase behavior of cationic hydroxyethyl cellulose-sodium dodecyl sulfate mixtures: Effects of molecular weight and ethylene oxide side chain length of polymers," Langmuir 20, 8482-8489 (2004).

${ }^{13}$ S. Trabelsi, S. Guillot, E. Raspaud, M. Delsanti, D. Langevin, and F. Boué, "New nano- and microparticles with a liquid-crystal-like interior," Adv. Mater. 18, 2403-2406 (2006).

${ }^{14}$ S. Dos Santos, C. Gustavsson, C. Gudmundsson, P. Linse, and L. Piculell, "When do water-insoluble polyion-surfactant ion complex salts 'redissolve' by added excess surfactant?," Langmuir 27, 592-603 (2010).

${ }^{15}$ V. D. Lam and L. M. Walker, "A pH-induced transition of surfactantpolyelectrolyte aggregates from cylindrical to string-of-pearls structure," Langmuir 26, 10489-10496 (2010).

${ }^{16}$ E. K. Penott-Chang, M. Ruppel, D. V. Pergushov, A. B. Zezin, and A. H. Müller, "Interpolyelectrolyte complexes of diblock copolymers via interaction of complementary polyelectrolyte-surfactant complexes in chloroform," Polymer 52, 4296-4302 (2011).

${ }^{17}$ E. Moroz, J. Zakharova, V. Sergeyev, and A. Zezin, "Ternary complexes polyelectrolyte-oppositely charged surfactant-carbon nanotubes: Formation and properties," Dokl. Phys. Chem. 441, 212-214 (2011).

${ }^{18}$ M. Štěpánek, J. Škvarla, M. Uchman, K. Procházka, B. Angelov, L. Kováčik, V. M. Garamus, C. Mantzaridis, and S. Pispas, "Wormlike core-shell nanoparticles formed by co-assembly of double hydrophilic block polyelectrolyte with oppositely charged fluorosurfactant," Soft Matter 8, 9412-9417 (2012).

${ }^{19}$ D. Li, M. S. Kelkar, and N. J. Wagner, "Phase behavior and molecular thermodynamics of coacervation in oppositely charged polyelectrolyte/surfactant systems: A cationic polymer JR 400 and anionic surfactant SDS mixture," Langmuir 28, 10348-10362 (2012).

${ }^{20}$ L. Chiappisi, I. Hoffmann, and M. Gradzielski, "Complexes of oppositely charged polyelectrolytes and surfactants-recent developments in the field of biologically derived polyelectrolytes," Soft Matter 9, 3896-3909 (2013). 
${ }^{21}$ C. G. de Kruif, J. S. Pedersen, T. Huppertz, and S. G. Anema, "Coacervates of lactotransferrin and beta- or kappa-casein: Structure determined using SAXS," Langmuir 29, 10483-10490 (2013).

${ }^{22} \mathrm{D}$. Li and N. J. Wagner, "Universal binding behavior for ionic alkyl surfactants with oppositely charged polyelectrolytes," J. Am. Chem. Soc. 135, 17547-17555 (2013).

${ }^{23}$ J. Hajduová, K. Procházka, M. Šlouf, B. Angelov, G. Mountrichas, S. Pispas, and M. Štepánek, "Polyelectrolyte-surfactant complexes of poly[3,5bis(dimethylaminomethyl)-4-hydroxystyrene]-block-poly(ethylene oxide) and sodium dodecyl sulfate: Anomalous self-assembly behavior," Langmuir 29, 5443-5449 (2013).

${ }^{24}$ L. Chiappisi, D. Li, N. J. Wagner, and M. Gradzielski, "An improved method for analyzing isothermal titration calorimetry data from oppositely charged surfactant polyelectrolyte mixtures," J. Chem. Thermodyn. 68, 48-52 (2014)

${ }^{25}$ T. Yin, M. Qin, Y. Yang, P. Zheng, D. Fan, and W. Shen, "The interactions of iota-carrageenan with cationic surfactants in aqueous solutions," Soft Matter 10, 4126-4136 (2014).

${ }^{26}$ L. Chiappisi, S. Prevost, I. Grillo, and M. Gradzielski, "Chitosan/alkylethoxy carboxylates: A surprising variety of structures," Langmuir 30, 1778-1787 (2014).

${ }^{27}$ A. Abraham, A. Kardos, A. Mezei, R. A. Campbell, and I. Vargat, "Effects of ionic strength on the surface tension and nonequilibrium interfacial characteristics of poly(sodium styrenesulfonate)/dodecyltrimethylammonium bromide mixtures," Langmuir 30, 4970-4979 (2014).

${ }^{28}$ L. Chiappisi, S. Prevost, and M. Gradzielski, "Form factor of cylindrical superstructures composed of globular particles," J. Appl. Crystallogr. 47, $827-834$ (2014).

${ }^{29}$ I. Hoffmann, "Neutrons for the study of dynamics in soft matter systems," Colloid Polym. Sci. 292, 2053-2069 (2014).

${ }^{30} \mathrm{E}$. Fegyver and R. Meszaros, "The impact of nonionic surfactant additives on the nonequilibrium association between oppositely charged polyelectrolytes and ionic surfactants," Soft Matter 10, 1953-1962 (2014).

${ }^{31}$ A. Bahramian, R. K. Thomas, and J. Penfold, "The adsorption behavior of ionic surfactants and their mixtures with nonionic polymers and with polyelectrolytes of opposite charge at the air-water interface," J. Phys. Chem. B 118, 2769-2783 (2014).

${ }^{32}$ S. Aidarova, A. Sharipova, J. Kragel, and R. Miller, "Polyelectrolyte/surfactant mixtures in the bulk and at water/oil interfaces," Adv. Colloid Interface Sci. 205, 87-93 (2014).

${ }^{33}$ Polymer-Surfactant Systems, edited by J. C. T. Kwak (Marcel Dekker, Inc., 1998), Vol. 77.

${ }^{34}$ C. D. Bain, P. M. Claesson, D. Langevin, R. Meszaros, T. Nylander, C. Stubenrauch, S. Titmuss, and R. von Klitzing, "Complexes of surfactants with oppositely charged polymers at surfaces and in bulk," Adv. Colloid Interface Sci. 155, 32-49 (2010).

${ }^{35}$ A. Mohr, T. Nylander, L. Piculell, B. Lindman, V. Boyko, F. W. Bartels, Y. Liu, and V. Kurkal-Siebert, "Mixtures of cationic copolymers and oppositely charged surfactants: Effect of polymer charge density and ionic strength on the adsorption behavior at the silica-aqueous interfaces," ACS Appl. Mater. Interfaces 4, 1500-1511 (2012).

${ }^{36}$ J. S. Weston, J. H. Harwell, B. J. Shiau, and M. Kabir, "Disrupting admicelle formation and preventing surfactant adsorption on metal oxide surfaces using sacrificial polyelectrolytes," Langmuir 30, 6384-6388 (2014).

${ }^{37}$ U. Kästner, H. Hoffmann, R. Donges, and R. Ehrler, "Interactions between modified hydroxyethyl cellulose (hec) and surfactants," Colloids Surf., A 112, 209-225 (1996).

${ }^{38}$ M. Tsianou, A. L. Kjøniksen, K. Thuresson, and B. Nyström, "Light scattering and viscoelasticity in aqueous mixtures of oppositely charged and hydrophobically modified polyelectrolytes," Macromolecules 32, 2974-2982 (1999).

${ }^{39} \mathrm{M}$. Tsianou and P. Alexandridis, "Control of the rheological properties in solutions of a polyelectrolyte and an oppositely charged surfactant by the addition of cyclodextrins," Langmuir 15, 8105-8112 (1999).

${ }^{40}$ I. S. Chronakis and P. Alexandridis, "Rheological properties of oppositely charged polyelectrolytesurfactant mixtures: Effect of polymer molecular weight and surfactant architecture," Macromolecules 34, 5005-5018 (2001).

${ }^{41}$ S. Guillot, D. McLoughlin, N. Jain, M. Delsanti, and D. Langevin, "Polyelectrolyte-surfactant complexes at interfaces and in bulk," J. Phys.: Condens. Matter 15, S219-S224 (2003).

${ }^{42}$ L. G. Patruyo, A. J. Müller, and A. E. Sáez, "Shear and extensional rheology of solutions of modified hydroxyethyl celluloses and sodium dodecyl sulfate," Polymer 43, 6481-6493 (2002).
${ }^{43}$ N. Jain, S. Trabelsi, S. Guillot, D. McLoughlin, D. Langevin, P. Letellier, and M. Turmine, "Critical aggregation concentration in mixed solutions of anionic polyelectrolytes and cationic surfactants," Langmuir 20, 8496-8503 (2004).

${ }^{44}$ N. Beheshti, G. T. M. Nguyen, A. L. Kjøniksen, K. D. Knudsen, and B. Nyström, "Structure and dynamics of aqueous mixtures of an anionic cellulose derivative and anionic or cationic surfactants," Colloids Surf., A 279, 40-49 (2006).

${ }^{45}$ Q. Wu, Y. Shangguan, M. Du, J. Zhou, Y. Song, and Q. Zheng, "Steady and dynamic rheological behaviors of sodium carboxymethyl cellulose entangled semi-dilute solution with opposite charged surfactant dodecyltrimethylammonium bromide," J. Colloid Interface Sci. 339, 236-242 (2009).

${ }^{46}$ I. Hoffmann, P. Heunemann, S. Prévost, R. Schweins, N. J. Wagner, and M. Gradzielski, "Self-aggregation of mixtures of oppositely charged polyelectrolytes and surfactants studied by rheology, dynamic light scattering and small-angle neutron scattering," Langmuir 27, 4386-4396 (2011).

${ }^{47}$ I. Hoffmann, S. Prévost, M. Medebach, S. E. Rogers, N. J. Wagner, and M. Gradzielski, "Control of rheological behaviour with oppositely charged polyelectrolyte surfactant mixtures," Tenside, Surfactants, Deterg. 48, 488-494 (2011).

${ }^{48}$ R. C. W. Liu, Y. Morishima, and F. M. Winnik, "Rheological properties of mixtures of oppositely charged polyelectrolytes. A study of the interactions between a cationic cellulose ether and a hydrophobically modified poly[sodium 2-(acrylamido)-2-methylpropanesulfonate]," Polym. J. 34, 340-346 (2002).

${ }^{49}$ I. Hoffmann, B. Farago, R. Schweins, P. Falus, M. Sharp, and M. Gradzielski, "Structure and dynamics of polyelectrolytes in viscous polyelectrolytesurfactant complexes at the mesoscale," Europhys. Lett. 104, 28001 (2013).

${ }^{50} \mathrm{U}$. Keiderling, "The new 'bersans-pc' software for reduction and treatment of small angle neutron scattering data," Appl. Phys. A: Mater. Sci. Process. 74, S1455-S1457 (2002).

${ }^{51}$ Neutrons, X-rays and Light: Scattering Methods Applied to Soft Condensed Matter, edited by P. Lindner and T. Zemb (Elsevier Science Ltd., 2002).

${ }^{52}$ P. Schleger, B. Alefeld, J. Barthelemy, G. Ehlers, B. Farago, P. Giraud, C. Hayes, A. Kollmar, C. Lartigue, F. Mezei, and D. Richter, "The longwavelength neutron spin-echo spectrometer IN15 at the Institut LaueLangevin," Phys. B 241-243, 164-165 (1997).

${ }^{53}$ F. Mezei, in Neutron Spin Echo, Lecture Notes in Physics edited by F. Mezei, (Springer, Berlin, Heidelberg, 1980), Vol. 128, pp. 1-26.

${ }^{54} \mathrm{~K}$. Thuresson, S. Nilsson, and B. Lindman, "Effect of hydrophobic modification on phase behavior and rheology in mixtures of oppositely charged polyelectrolytes," Langmuir 12, 530-537 (1996).

${ }^{55}$ See supplementary material at http://dx.doi.org/10.1063/1.4928583 for more information on the polymers, the exact composition of samples and additional data.

${ }^{56}$ J. Hayter, G. Janninck, F. Brochardwyart, and P. G. de Gennes, "Correlations and dynamics of poly-electrolyte solutions," J. Phys., Lett. 41, L451-L454 (1980).

${ }^{57}$ F. Nallet, G. Jannink, J. B. Hayter, R. Oberthur, and C. Picot, “Observation of the dynamics of poly-electrolyte strong solutions by quasi-elastic neutronscattering," J. Phys. (Paris) 44, 87-99 (1983).

${ }^{58}$ A. Z. Akcasu, M. Benmouna, and B. Hammouda, "On the dynamics of polyelectrolyte solutions," J. Chem. Phys. 80, 2762-2766 (1984).

${ }^{59}$ S. Lee, V. R. Tirumala, M. Nagao, T. Tominaga, E. K. Lin, J. P. Gong, and W.-L. Wu, "Dynamics in multicomponent polyelectrolyte solutions," Macromolecules 42, 1293-1299 (2009).

${ }^{60}$ M. Adam, D. Lairez, E. Raspaud, and B. Farago, "Dynamic properties of semidilute solutions at the theta point," Phys. Rev. Lett. 77, 3673-3676 (1996).

${ }^{61}$ A. Akcasu, M. Benmouna, and C. C. Han, "Interpretation of dynamic scattering from polymer solutions," Polymer 21, 866-890 (1980).

${ }^{62}$ A. Arbe, M. Monkenbusch, J. Stellbrink, D. Richter, B. Farago, K. Almdal, and R. Faust, "Origin of internal viscosity effects in flexible polymers: A comparative neutron spin-echo and light scattering study on poly(dimethylsiloxane) and polyisobutylene," Macromolecules 34, 1281-1290 (2001).

${ }^{63} \mathrm{C}$. Scherzinger, O. Holderer, D. Richter, and W. Richtering, "Polymer dynamics in responsive microgels: Influence of cononsolvency and microgel architecture," Phys. Chem. Chem. Phys. 14, 2762-2768 (2012).

${ }^{64}$ E. Dubois-Violette and P. G. de Gennes, "Quasi-elastic scattering by dilute ideal polymer solutions. 2. Effects of hydrodynamic interactions," Phys. (New York) 3, 181 (1967). 
${ }^{65}$ B. H. Zimm, "Dynamics of polymer molecules in dilute solution: Viscoelasticity, flow birefringence and dielectric loss," J. Chem. Phys. 24, 269-278 (1956).

${ }^{66}$ B. D. Ermi and E. J. Amis, "Influence of backbone solvation on small angle neutron scattering from polyelectrolyte solutions," Macromolecules 30, 6937-6942 (1997).

${ }^{67}$ B. D. Ermi and E. J. Amis, "Domain structures in low ionic strength polyelectrolyte solutions," Macromolecules 31, 7378-7384 (1998).

${ }^{68}$ J. Ray and G. S. Manning, "Formation of loose clusters in polyelectrolyte solutions," Macromolecules 33, 2901-2908 (2000).

${ }^{69}$ B. Lindman, G. Karlström, and L. Stigsson, "On the mechanism of dissolution of cellulose," J. Mol. Liq. 156, 76-81 (2010).

${ }^{70}$ B. Medronho, A. Romano, M. Miguel, L. Stigsson, and B. Lindman, "Rationalizing cellulose (in)solubility: Reviewing basic physicochemical aspects and role of hydrophobic interactions,' Cellulose 19, 581-587 (2012).
${ }^{71}$ J. Merta, V. M. Garamus, R. Willumeit, and P. Stenius, "Structure of complexes formed by pdadmac and sodium palmitate," Langmuir 18, 7272-7278 (2002).

${ }^{72}$ N. A. Mazer, G. B. Benedek, and M. C. Carey, "An investigation of the micellar phase of sodium dodecyl sulfate in aqueous sodium chloride solutions using quasielastic light scattering spectroscopy," J. Phys. Chem. 80, 1075-1085 (1976).

${ }^{73}$ D. C. Cheng and E. Gulari, "Micellization and intermicellar interactions in aqueous sodium dodecyl benzene sulfonate solutions," J. Colloid Interface Sci. 90, 410-423 (1982).

${ }^{74} \mathrm{G}$. Manning, "Counterion condensation theory of attraction between like charges in the absence of multivalent counterions," Eur. Phys. J. E 34, 132 (2011).

${ }^{75}$ S. Broersma, "Rotational diffusion constant of a cylindrical particle," J. Chem. Phys. 32, 1626-1631 (1960). 